\title{
ON THE INTERSECTION OF VARIETIES WITH A TOTALLY REAL SUBMANIFOLD
}

\author{
HOWARD JACOBOWITZ
}

\begin{abstract}
In their work on uniqueness in the Cauchy problem for CR functions, Baouendi and Treves have utilized a condition on a totally real submanifold $M$ in a neighborhood of one of its points $p$ : There should exist a variety $X$ such that the component containing $p$ of $M-(M \cap X)$ has compact closure in $M$. All real analytic submanifolds satisfy this condition. In this paper, a $C^{\infty}$ submanifold is constructed which does not. Uniqueness in the corresponding Cauchy problem remains unresolved.
\end{abstract}

The generic intersection of two submanifolds of $\mathbf{R}^{4}$, each of dimension two, is a single point. Of course in any particular case other intersections are possible. For instance, the totally real submanifold $\left\{\left(z_{1}, z_{2}\right): z_{1}\right.$ and $z_{2}$ are real $\}$ of $\mathbf{C}^{2}$ intersects the complex curve $\left\{\left(z_{1}, z_{2}\right): z_{1}^{2}+z_{2}^{2}=1\right\}$ in a circle. From this we may see that for any totally real, real analytic surface $M^{2}$ in $\mathbf{C}^{2}$ which contains the origin and for any neighborhood $V$ of the origin there exists a smaller neighborhood $U$ and a function $F$ holomorhic on $U$ such that $M^{2} \cap F^{-1}(0)$ contains a closed curve about the origin.

The following condition has been shown by Baouendi and Treves to imply a uniqueness result for CR functions [BT]. We state this condition as it arises in the uniqueness result for four-dimensional $\mathrm{CR}$ submanifolds in $\mathbf{C}^{3}$. Here $\Sigma$ is a totally real two-dimensional manifold in $\mathbf{C}^{2}$ and $p$ is some point of $\Sigma$.

Condition A. Given any sufficiently small neighborhood $V$ of $p$ in $\Sigma$ there is a function $F$ holomorphic in an open set of $\mathbf{C}^{2}$ containing $V$ such that $F(p) \neq 0$ and the connected component of $p$ in the set $V \cap\{q: F(q) \neq 0\}$ has compact closure contained in $V$.

Note that if for a particular $V$ such a function $F$ exists, then $F$ also suffices for a somewhat smaller $V^{\prime}$ and now $F$ is holomorphic in a neighborhood of $\overline{V^{\prime}}$. Hence to provide a $\Sigma$ which does not satisfy Condition A we need only show that $\Sigma$ does not satisfy the somewhat more restrictive condition where "containing $V$ " is replaced by "containing $\bar{V}$."

By the remarks above, Condition A and hence also the uniquenes result in [BT] hold when $\Sigma$ is real analytic. In this paper we construct a $\Sigma$ of class $C^{\infty}$ for which Condition A fails. It is not known if the uniqueness result also fails. In our proof that Condition $\mathrm{A}$ is not valid for this particular $\Sigma$, we use the following two lemmas.

Received by the editors May 28, 1986.

1980 Mathematics Subject Classification (1985 Revision). Primary 32B99; Secondary 35F10.

Key words and phrases. Cauchy problem for CR functions, totally real submanifolds.

This research was partially supported by NSF Grant DMS 8402631. It is a pleasure to acknowledge that this version of the example was worked out during a visit to Purdue University and that various improvements emerged in conversations with M. S. Baouendi. 
LEMMA 1. Let $U$ and $U_{1}$ be open sets in $\mathbf{C}^{2}$ with $\bar{U} \subset U_{1}$ and let $F$ be a holomorphic function on $U_{1}, F$ not identically zero on any component of $U$. Then for each $q \in U \cap F^{-1}(0)$, except for finitely many, there exists an open neighborhood $U_{q}$ of $q$ and a function $F_{q}$ holomorphic on $U_{q}$ such that

(a) $F_{q}$ is either of the form $z_{1}-f\left(z_{2}\right)$ or $z_{2}-f\left(z_{1}\right)$,

(b) $U_{q} \cap F^{-1}(0)=U_{q} \cap F_{q}^{-1}(0)$.

PROOF. Recall that a point $q$ on the variety $F^{-1}(0)$ is called a regular point if there is a neighborhood $V_{q}$ of $q$ such that $F^{-1}(0) \cap V_{q}$ is a complex submanifold of $V_{q}$ [GR]. On some smaller neighborhood $U_{q}$, this submanifold must be given either by $z_{1}=f\left(z_{2}\right)$ or $z_{2}=f\left(z_{1}\right)$. Thus we need show that there can only be a finite number of singular points of $F^{-1}(0) \cap U$. This is true because the singular points form a variety of dimension less than that of $F^{-1}(0)$ [W, p. 92] and, as is easily seen, any variety of dimension zero is a discrete set of points.

LEMMA 2. Let $F$ be a complex valued, real analytic function near the origin in $\mathbf{R}^{2}$. There exist a neighborhood $U$ of the origin and an integer $N$ such that on each line segment $\{(x, y): y=c\} \cap U$ either $F$ has, less than $N$ zeros or $F$ is identically zero.

This follows from, for instance, the much stronger results in $[\mathbf{H}]$.

The totally real two-dimensional submanifold $\Sigma$ of $\mathbf{C}^{2}$ which we seek will be of the form

$$
\Sigma=\left\{\left(z_{1}, z_{2}\right): z_{1}=x_{1}, z_{2}=x_{2}+i \phi\left(x_{1}\right)\right\},
$$

where $\phi$ is a $C^{\infty}$ function which we now describe. Let $p_{j}=1 / j$ and let $\left\{B_{j, k}\right\}$ be a set of disjoint intervals with $B_{j, k}$ converging to the point $p_{j}$ as $k$ goes to infinity. Let $\phi \in C^{\infty}(\mathbf{R})$ be positive in each $B_{j, k}$, zero at each $p_{j}$, and with supp $\phi=\overline{\bigcup_{j, k} B_{j, k}}$. Let $S_{j, k}=\left\{\left(x_{1}, x_{2}\right): x_{1} \in B_{j, k}\right\}$ and $l_{j}=\left\{\left(x_{1}, x_{2}\right): x_{1}=p_{j}\right\}$. Then as a function on $\mathbf{R}^{2}$, supp $\phi=\overline{\bigcup_{j, k} S_{j, k}}$. Note that for any $j$ and any integer $N$ there exists an $\varepsilon$ such that $\phi(x)=\mu$ has more than $N$ solutions in $\bigcup_{k} B_{j, k}$ provided $0<\mu<\varepsilon$. Also note that $\Sigma$ is totally real because at any point $p \in \Sigma$ the tangent space $T_{p} \Sigma$ is spanned by $\partial / \partial x_{2}$ and $\partial / \partial x_{1}+\phi^{\prime}\left(x_{1}\right) \partial / \partial y_{2}$, and so $T_{p} \Sigma \cap J\left(T_{p} \Sigma\right)=\{0\}$ for the complex structure operator $J$.

THEOREM. Let $V$ be any neighborhood of the origin in $\Sigma$ and $F$ any function holomorphic in a open set of $\mathbf{C}^{2}$ containing $\bar{V}$ with $F(0) \neq 0$. There exists a piecewise smooth curve $\gamma(t)$ in $\Sigma$ with $\gamma(0)=0, \gamma(1) \in \partial V$, and $F(\gamma(t))$ different from zero for each $t$ in $0 \leq t \leq 1$.

PrOOF. We may replace $V$ by its connected component containing the origin. Let $F$ be holomorphic on some $U_{1}$ and let $U$ be a connected open set with $\bar{V} \subset U \subset$ $\bar{U} \subset U_{1}$. We apply Lemma 1 to find some $j$ for which $l_{j}$ does not contain any of the finite number of points excluded in this lemma. We also take $j$ large enough so that $F(x, 0) \neq 0$ for $x$ satisfying $0 \leq x \leq 1 / j$. In particular $F$ cannot be identically zero on the component of $l_{j} \cap U$ which contains $(1 / j, 0)$ and so $F$ can have only a finite number of zeros on the component of $l_{j} \cap \bar{V}$ which contains this point. If we enlarge $V$ a little then we also have $F$ is different from zero at the two endpoints of this component. (We may enlarge $V$, since if the theorem holds for some $V^{\prime}$ with 
$\bar{V} \subset V^{\prime}$, then it clearly also holds for $V$.) For this choice of $j$ let $p$ be the point $(1 / j, 0), l$ the line $l_{j}$, and $S_{k}$ the strip $S_{j, k}$. To summarize, we now have

(a) $F$ has only finitely many zeros on the component of $l \cap V$ which contains $p$;

(b) near each of these zeros, the zero set of $F$ in $\mathbf{C}^{2}$ is given by either $z_{1}=f\left(z_{2}\right)$ or $z_{2}=f\left(z_{1}\right)$; and

(c) $F \neq 0$ at the two boundary points of this component of $l$.

Since there is a curve in $\Sigma$ from the origin to $p$ on which $F$ is not zero, it suffices to find such a curve from $p$ to one of the boundary points of this component of $l$. This curve will proceed along $l$ until it approaches a zero of $F$. The next lemma is used to avoid this zero along a curve which returns to $l$ beyond this zero. A finite number of such detours then provides the curve $\gamma(t)$ in the theorem. For convenience we introduce new coordinates so that $l$ is the $x_{2}$-axis, $p$ is the origin, and $I$ is the line segment $I=\{(0, s): 0 \leq s<1\}$ which lies in $V$ with endpoint $(0,1)$ on $\partial V$. We have $F$ holomorphic in a neighborhood of $I ; F(0,0) \neq 0 ; F(0,1) \neq 0$; and $F$ has only a finite number of zeros on $I$. Let $q=(0, s)$ be any one of these zeros and for some small $\delta$ take numbers $s_{-}$and $s_{+}$with $s-\delta<s_{-}<s<s_{+}<s+\delta$. Let $q_{ \pm}=\left(0, s_{ \pm}\right)$.

LEMMA 3. If $\delta$ is small enough, there exists a piecewise smooth curve $\sigma(t)$ in $\Sigma$ with $\sigma(0)=q_{-}, \sigma(1)=q_{+}$, and $F(\sigma(t))$ different from zero for each $t$ in $0 \leq t \leq 1$.

PROOF. Find an $\varepsilon$ such that in the ball $B(q, \varepsilon)$ the zero set of $F$ is given by

(i) $z_{1}=f\left(z_{2}\right)$ or

(ii) $z_{2}=f\left(z_{1}\right)$.

Now choose $\varepsilon_{1}$ and $\delta$ so small that $B\left(q_{-}, \varepsilon_{1}\right)$ and $B\left(q_{+}, \varepsilon_{1}\right)$ are contained in $B(q, \varepsilon)$. Let $D$ be the "rectangle" in $\Sigma$ given by

$$
D=\left\{x_{1}, x_{2}+i \phi\left(x_{1}\right): 0 \leq x_{1} \leq \varepsilon_{1}, s_{-} \leq x_{2} \leq s_{+}\right\} .
$$

Thus $D$ is bounded by a segment of $l$, a line segment parallel to $l$, and by curves passing through $q_{-}$and $q_{+}$. Note that $D \subset B(q, \varepsilon)$ and so one of the forms (i) or (ii) is valid throughout $D$. Also note that as long as $\varepsilon_{1}$ is small enough we have that $F$ is nonzero on the top and bottom boundaries of $D$. Thus, to prove the lemma, we need only find a segment in $D$ parallel to $l$ along which $F$ is never zero.

The proof that such a segment exists is divided into two cases corresponding to the equations $z_{1}=f\left(z_{2}\right)$ and $z_{2}=f\left(z_{1}\right)$ for the zero set.

Case (i). The zero set of $F$ is given by $z_{1}=f\left(z_{2}\right)$. Let $v\left(x_{2}, y_{2}\right)=\operatorname{Im} f\left(x_{2}+i y_{2}\right)$. We claim that $v$ is not identically zero. This is so because if it were, then $f\left(z_{2}\right)$ would be a constant but $f(s)=0$ and $f\left(s_{-}\right) \neq 0$. Thus there exists a sequence of real numbers $\mu_{k} \rightarrow 0$ such that $v\left(x_{2}, \mu_{k}\right)$ is not identically zero on the interval $s_{-} \leq x_{2} \leq s_{+}$. Lemma 2 then guarantees that for some integer $N$ each equation $v\left(x_{2}, \mu_{k}\right)=0$, for $k$ large, can have no more than $N$ solutions $x_{2}=x_{2}\left(\mu_{k}\right)$ in this interval. (The application of this lemma might necessitate shrinking $\varepsilon$, and hence also $\varepsilon_{1}$ and $\delta$.) Clearly we may take each $\mu_{k}$ to be positive. We want to show that $F$ is everywhere different from zero for some segment in $D$. We reason by contradiction and so start with the assumption that for each $x_{1}$ there is some $x_{2}$ in $s_{-} \leq x_{2} \leq s_{+}$such that $F$ has a zero at the point $\left(x_{1}, x_{2}+i \phi\left(x_{1}\right)\right)$. Thus for each $x_{1}$ in $0 \leq x_{1} \leq \varepsilon_{1}$ there is at least one $x_{2}$ in $s_{-} \leq x_{2} \leq s_{+}$with $x_{1}=f\left(x_{2}+i \phi\left(x_{1}\right)\right)$. For each $\mu$ let $N(\mu)$. Jenote the number of solutions to $\phi\left(x_{1}\right)=\mu$ in $0 \leq x_{1} \leq \varepsilon_{1}$. 
As we have already noted, $N(\mu) \rightarrow \infty$ as $\mu \rightarrow 0$ with $\mu>0$. Thus the equation $x_{1}=f\left(x_{2}+i \mu\right)$ has $N(\mu)$ solution pairs $\left(x_{1}, x_{2}\right)$. Further, no two solution pairs with different values of $x_{1}$ can have the same $x_{2}$. Thus along the line segment in the $z_{2}$-plane given by $s_{-} \leq \operatorname{Re} z_{2} \leq s_{+}$and $\operatorname{Im} z_{2}=\mu$, the holomorphic function $f$ is real for at least $N(\mu)$ points. But then $N(\mu) \rightarrow \infty$ contradicts the existence of the bound $N$ for solutions to $\operatorname{Im} f\left(x_{2}+i \mu_{k}\right)=0$.

Case (ii). The zero set of $F$ is given by $z_{2}=f\left(z_{1}\right)$. If $F$ does not have a zero on some segment $\left\{\left(x_{1}, x_{2}\right): x_{1}=c \notin \operatorname{supp} \phi, s_{-} \leq x_{2} \leq s_{+}\right\}$, then we are done. So we now show that if $F$ does have a zero on each such segment then $F$ cannot have any zeros in the interior of the support of $\phi$ and thus any segment in this set provides the desired curve. So let us assume that for each $x_{1} \notin \operatorname{supp} \phi$ there is some $x_{2}$ with $x_{2}=f\left(x_{1}\right)$. Thus $f$ is real valued on an interval of the real axis. Hence it is real valued on the entire piece of the $x_{1}$-axis in $B(q, \varepsilon)$. Thus $x_{2}+i \phi\left(x_{1}\right)=f\left(x_{1}\right)$ can be satisfied only when $\phi\left(x_{1}\right)=0$. Hence $f$ has no zeros in $\left\{\left(x_{1}, x_{2}+i \phi\left(x_{1}\right)\right): \phi\left(x_{1}\right) \neq 0, s_{-} \leq x_{2} \leq s_{+}\right\}$.

\section{REFERENCES}

[BT] M. S. Baouendi and F. Treves, Unique continuation in CR manifolds and in hypo-analytic structures, Amer. J. Math. (to appear).

[GR] R. C. Gunning and H. Rossi, Analytic functions of several complex variables, Prentice-Hall, Englewood Cliffs, N.J., 1965.

[H] R. Hardt, Slicing and intersection theory for chains associated with real analytic varieties, Acta Math. 129 (1972), 75-136.

[W] H. Whitney, Complex analytic varieties, Addison-Wesley, Reading, Mass., 1972.

Department of Mathematics, Rutgers University, Camden, New Jersey 08102 